\title{
POLYMER SPREADING ON UNIDIRECTIONALLY NANOTEXTURED SUBSTRATES USING MOLECULAR DYNAMICS
}

\author{
Brooklyn A. Noble and Bart Raeymaekers
}

Department of Mechanical Engineering, University of Utah, Salt Lake City, UT 84112, USA

\section{Potential function interactions}

Figure S1 depicts the potential function interactions that define the molecular dynamics simulations described in this work. The potentials are identified using arrows and numbers that correspond to the equations that describe the potentials, which are similar to validated potentials and parameters used in previous research.

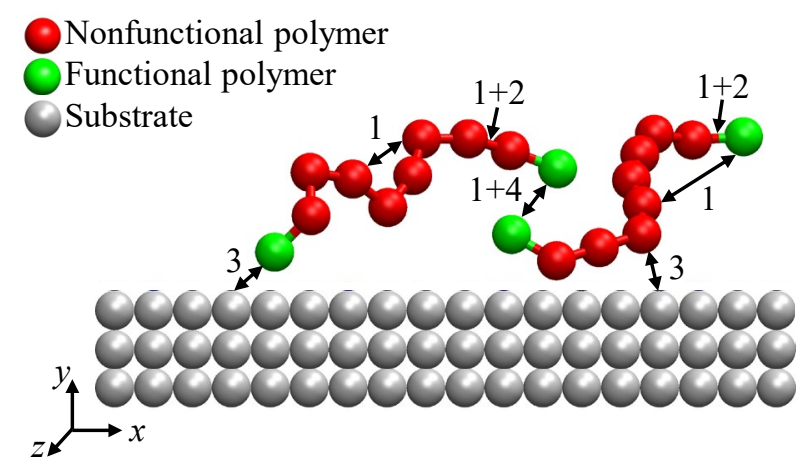

Figure S1. Schematic of energy potential function interactions where numbers refer to the corresponding equations.

A Lennard Jones potential $U_{L J}$ exists between all polymer beads, given by

$$
U_{L J}(r)=4 \varepsilon\left[\left(\frac{\sigma}{r}\right)^{12}-\left(\frac{\sigma}{r}\right)^{6}-\left(\frac{\sigma}{r_{c}}\right)^{12}+\left(\frac{\sigma}{r_{c}}\right)^{6}\right]
$$

where $r$ is the distance between two beads, $\sigma=0.7 \mathrm{~nm}$ is the bead diameter, $r_{c}=2.5 \sigma$ is the cutoff distance within which interactions between neighboring beads are considered, and $\varepsilon=T k_{B}$ is the potential well depth, where $T=300 \mathrm{~K}$ is the absolute temperature and $k_{B}$ is the Boltzmann constant. A finitely extensible 
nonlinear elastic (FENE) potential $U_{F E N E}$ acts as a spring to bond neighboring beads of the same molecule, i.e.,

$$
U_{F E N E}(l)=-\frac{1}{2} k R_{0}^{2} \ln \left[1-\left(\frac{l-l_{0}}{R_{0}}\right)^{2}\right],
$$

where $l$ is the bond length, $l_{0}=1.3 \sigma$ is the equilibrium bond length, $R_{0}=0.3 \sigma$ indicates the maximum extensible range, and $k=40 \varepsilon / \sigma^{2}$ is the spring constant. The substrate interacts with the polymer via a dispersive van der Waals interaction $U_{V D W}$, described as

$$
U_{V D W}(r)=2 \varepsilon\left[\frac{1}{2}\left(\frac{\sigma}{r}\right)^{9}-\frac{3}{2}\left(\frac{\sigma}{r}\right)^{3}\right] .
$$

We also use a short-range exponential potential $U_{E X P}$ to model the attraction of functional end beads to each other, which represents the attraction of functional hydroxyl groups and is given by

$$
U_{E X P}(r)=-2 \varepsilon \exp \left(-\frac{r-r_{c}}{d}\right)
$$

where $r_{c}=1.0 \sigma$ is the cutoff distance and $d=0.3 \sigma$ is the decay length.

\section{Number of grooves and peaks encountered}

Figure S2 shows top views of polymer after spreading on substrates with $\theta=60^{\circ}$ peaks, $\theta=60^{\circ}$ grooves, and texture lengths: (a) $L=25 \sigma$, (b) $L=50 \sigma$, (c) $L=100 \sigma$, and (d) $L=250 \sigma$. Dashed red lines show the locations of texture peaks, solid blue lines shown the locations of texture grooves, and each polymer bead is shown as a small black dot. From Fig. S2 (a) with $L=25 \sigma$, we observe that the polymer encounters five grooves during spreading and we note that $d_{\perp}=242 \sigma$ is significantly smaller (approximately $35 \%$ decrease) than the isotropic spreading observed on a flat substrate $\left(d_{\perp}=372 \sigma\right)$. From Fig. S2 (b) with 
$L=50 \sigma$, we observe that the polymer encounters three grooves during spreading. Comparing to $L=25 \sigma$, $d_{\perp}=309 \sigma$ increases but remains less than the spreading observed on a flat substrate. From Fig. S2 (c) with $L=100 \sigma$, we observe that the polymer only encounters one groove, above which the polymer is initially placed. Thus, the polymer overcomes zero grooves but two peaks, and we observe that the peaks do not inhibit spreading because $d_{\perp}=367 \sigma$ is similar $(1.3 \%$ decrease $)$ to that obtained on a flat substrate. From Fig. S2 (d) with $L=250 \sigma$, we observe the polymer only encounters a single groove and no peaks. The polymer spreads in a single groove and $d_{\perp}$ is unaffected by the texture $\left(d_{\perp}=376 \sigma\right)$.
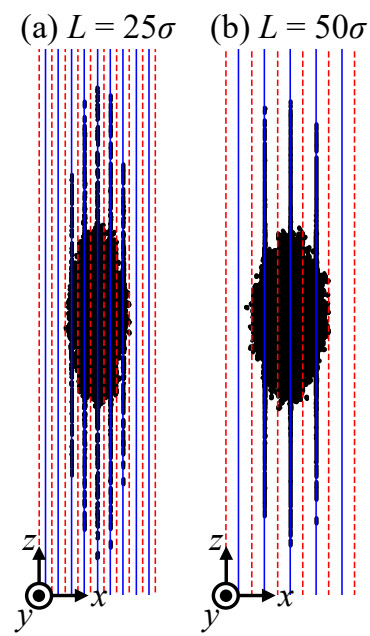

(c) $L=100 \sigma$

(d) $L=250 \sigma$
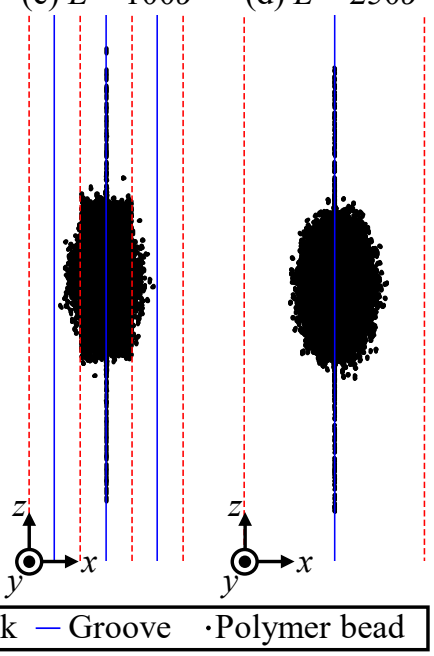

Figure S2. Top views of spread polymer on substrates with $\theta=60^{\circ}$ peaks, $\theta=60^{\circ}$ grooves, and texture lengths: (a) $L=25 \sigma$, (b) $L=50 \sigma$, (c) $L=100 \sigma$, and (d) $L=250 \sigma$. Dashed red lines show the locations of texture peaks, solid blue lines shown the locations of texture grooves, and each polymer bead is shown as a small black dot.

\section{Local polymer quantity}

Figure S3 shows 2D local polymer quantity maps at the peaks and grooves of the five different substrates shown in Fig. 4 of the manuscript. Fig. S3 (a) shows a flat substrate with a single polymer quantity map for reference. From Fig. S3, we observe dark red regions in texture grooves where polymer beads cluster to minimize their energy state. These dark red regions are most pronounced in Fig. S3 (b)-(d), which corresponds to the substrates with the smallest minimum potential energy. From Fig. S3, we also observe 
that the local polymer quantity locally decreases at texture peaks. Polymer molecules avoid these regions to minimize their energy state.
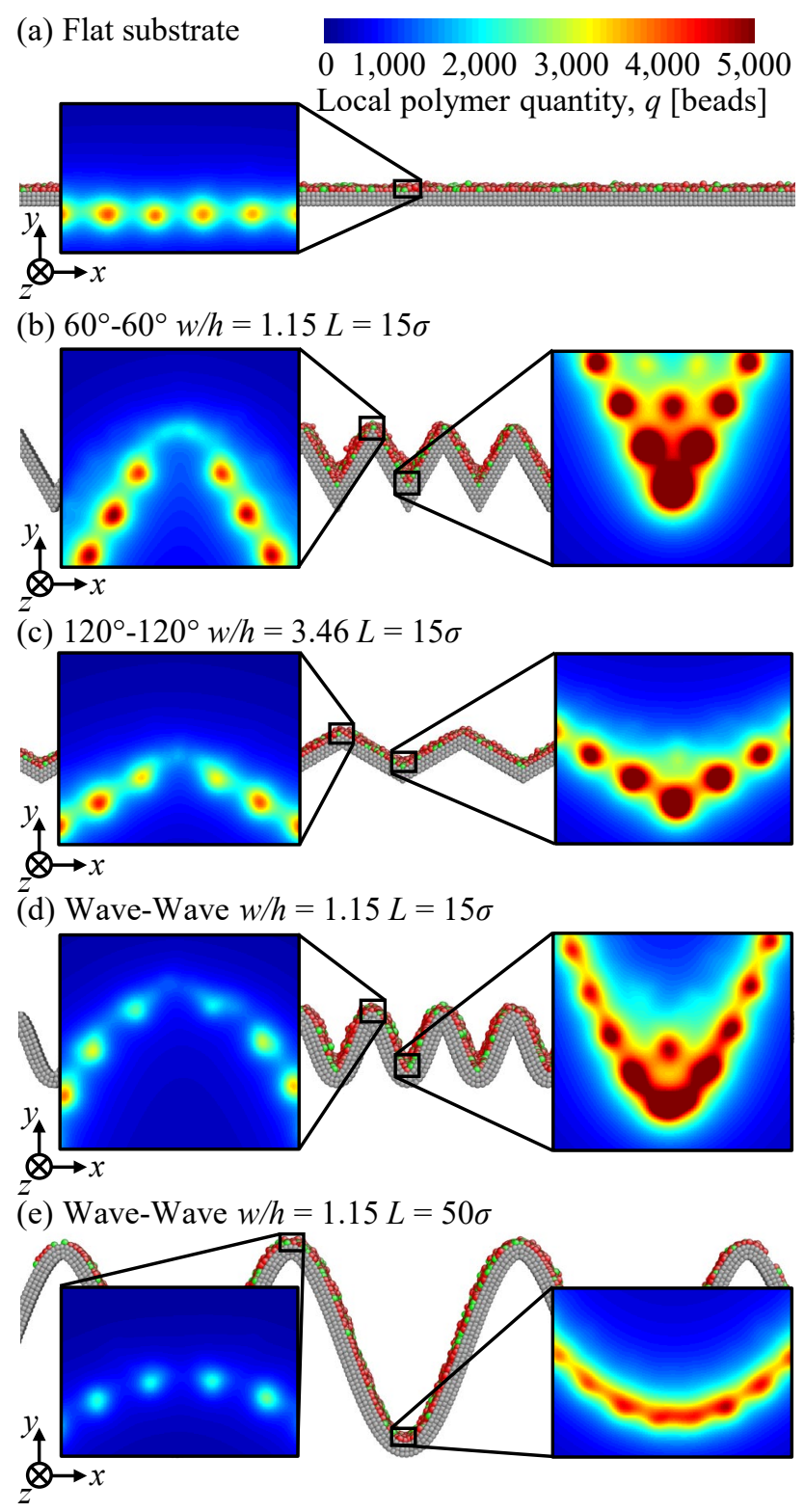

Figure S3. 2D local polymer quantity maps at the peaks and grooves of substrates shown in Fig. 4 of the manuscript with different texture shapes and lengths.

\section{Spreading and minimum potential energy correlation}

Figure S4 shows the polymer spreading parallel and perpendicular to the texture versus the texture minimum energy for substrates with texture length $L=15 \sigma$, corresponding to Fig. 5 (a). We report $R$ - 
squared values for the linear best fit (least-squares) to each data set ( $d_{\perp}$ and $\left.d_{\|}\right)$. From Fig. S4, we observe that parallel spreading correlates to the minimum potential energy and that perpendicular spreading inversely correlates to the minimum potential energy.

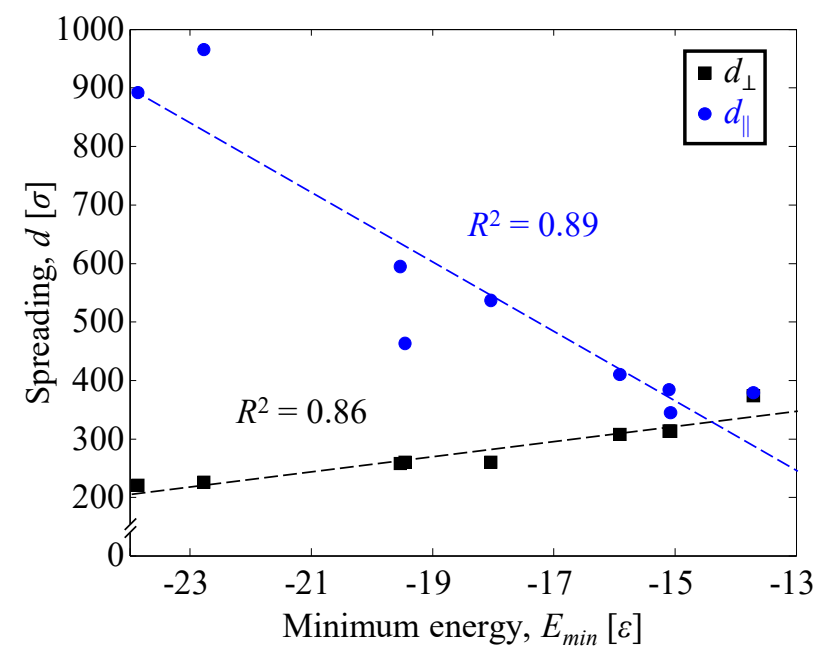

Figure S4. Polymer spreading parallel and perpendicular to the texture versus texture minimum energy for substrates with texture length $L=15 \sigma$. 